\title{
Automated oscillometric measurement of the ankle-brachial index in patients with coronary artery disease
}

\author{
Maciej Sinski, Grzegorz Styczynski and Cezary Szmigielski
}

Automated oscillometric ankle-brachial index ( $A B I)$ devices were designed to measure $A B I$ in a primary-care setting to increase the peripheral artery disease (PAD) detection rate. However, ABI measurements obtained with an automated oscillometric device may differ from those obtained using a standard ultrasound Doppler method in the general population. The purpose of this study was to compare PAD detection by the Doppler method and the automated WatchBP Office ABI system in a high-risk population with coronary artery disease (CAD). Eighty consecutive patients with confirmed CAD were included. ABI was measured by automated oscillometry followed by conventional Doppler evaluation. PAD was defined as an $A B I \leqslant 0.9$. Each lower extremity was analyzed separately. The Doppler method detected an $A B I \leqslant 0.9$ in 56 lower extremities, whereas the automated method detected an $A B I \leqslant 0.9$ in 28 lower extremities $(P<0.0001)$. A Bland-Altman plot showed poor agreement between the two methods. The mean $A B I$ values obtained by the automated and Doppler methods were significantly different $(1.11 \pm 0.20$ vs. $0.95 \pm 0.24 ; P<0.00001$ ). The sensitivity of the automated $A B I$ device in detecting an $A B I \leqslant 0.9$ was $46.3 \%$ and the specificity was $98.0 \%$. The positive and negative predictive values for diagnosing an $A B I \leqslant 0.9$ using the automated oscillometric method were $92.8 \%$ and $76.9 \%$, respectively. In conclusion, the automated WatchBP Office ABI system should be used with caution for PAD detection and screening in patients with CAD, and this system should not replace the Doppler method in populations at high risk of cardiovascular disease.

Hypertension Research (2013) 36, 25-28; doi:10.1038/hr.2012.120; published online 9 August 2012

Keywords: ankle-brachial index; automated oscillometric method; coronary artery disease; doppler method; peripheral artery disease

\section{INTRODUCTION}

Peripheral artery disease (PAD) remains undiagnosed in nearly half of all patients with this disease. ${ }^{1}$ Practitioners diagnose PAD mainly on the basis of a patient's medical history and physical examination. However, a substantial number of patients with PAD are asymptomatic, and the sensitivity of physical examination for PAD detection is low. ${ }^{2,3}$

Ankle-brachial index (ABI) measurement is an established method for detecting PAD. An abnormal ABI (value $\leqslant 0.9$ ) is sensitive and specific for detecting PAD. ${ }^{4-6}$ The Doppler method is the gold standard method of ABI measurement; however, it is relatively time consuming and is significantly operator-dependent. ${ }^{7}$ Thus, there is a need for an easier but reliable method for ABI determination. ABI measurement based on an oscillometric method has shown promising results. ${ }^{8}$ Automated oscillometric systems that measure blood pressure simultaneously on the upper and lower limbs were introduced to simplify the measurement of $\mathrm{ABI}$ and to make such measurements feasible for outpatient clinics and primary-care physicians. These automated systems are attractive due to the simplicity of the method and the short time needed to obtain results. However, data concerning the validity of the automated devices, compared with Doppler-based devices, have been inconsistent. Although some studies found a good correlation of automated systems with the Doppler method, ${ }^{9-11}$ other studies, including population studies, did not confirm those findings. ${ }^{12-15}$

The use of automated oscillometric ABI systems has not been studied extensively in a population with known atherosclerosis. ${ }^{16}$ Similarly, such data are limited in populations with very high cardiovascular risk. ${ }^{17}$

The purpose of our study was to compare the automated WatchBP Office ABI system with the reference standard Doppler method in a population of patients with known coronary artery disease (CAD).

\section{METHODS}

We studied 80 consecutive patients with diagnosed CAD. The diagnosis of CAD was based on one of the following criteria: the patient's history of previous myocardial infarction combined with regional wall motion abnormalities on echocardiography; significant findings on coronarography; history of coronary interventions (percutaneous coronary intervention (PCI) or coronary artery bypass graft (CABG)). The patients were enrolled into the 
study from the population referred to our department for an echocardiographic examination. The study was approved by the institution's ethics committee. All subjects gave informed consent before participation in the study.

Before the study procedures, each patient was in the supine position for 20-30 min while echocardiographic recording was performed. After echocardiography, an automated oscillometric $\mathrm{ABI}$ measurement was performed using the WatchBP Office ABI system (Microlife WatchBP AG, Widnau, Switzerland). The set includes three cuffs. Both Doppler measurements and automated oscillometric recordings were performed using appropriately sized cuffs. In the first procedure, blood pressure was measured simultaneously on both arms. The arm with the higher systolic blood pressure was selected for the ABI measurement. One of the brachial cuffs was then replaced with the ankle cuff. The ankle cuff was placed over the posterior tibial artery on the ankle. Both cuffs were inflated simultaneously, and the ABI was calculated automatically. The same measurement was performed on the other ankle. Patients with peripheral edema and atrial fibrillation at the time of the measurement were excluded from the study. The automated oscillometric measurement was performed by a single experienced technician according to the manual supplied with the device.

The Doppler ABI was measured within 5-10 min of the automated oscillometric measurement using a linear vascular probe with the ultrasound unit (GE Vivid 5, GE Vingmed, Horten, Norway) or Philips IE 33 (Philips Medical Systems, Andover, MA, USA) and a sphygmomanometer (Heine G5, Heine Optotechnik, Herrsching, Germany). The measurements were started by determining systolic blood pressure on the brachial arteries. A cuff was placed over the brachial artery and inflated $20 \mathrm{~mm} \mathrm{Hg}$ above systolic pressure and then released until the first signal of the Doppler flow was recorded. The higher systolic blood pressure was recorded for the ABI calculation. After the brachial artery measurements, systolic blood pressure was measured in the same way on both ankles. Specifically, a Doppler probe was placed over the posterior tibial artery, which was the site used for the automatic oscillometric measurement. All Doppler ABI measurements were performed by a single experienced physician blinded to the results of the automated oscillometric measurements.

The data are presented as means \pm s.d. The mean results of the two methods were compared using the Student's $t$-test for paired data. The McNemar test for proportions was used to determine differences in the detection of an $\mathrm{ABI} \leqslant 0.9$ by both methods. The Pearson's correlation coefficient was used to measure associations between the methods. Agreement between the methods was evaluated using Bland-Altman analysis. ${ }^{18}$ Sensitivity, specificity, positive predictive value and negative predictive value were calculated for the use of the WatchBP oscillometric ABI device to determine an $\mathrm{ABI} \leqslant 0.9$ using the Doppler method as the reference. The differences between the methods were plotted as a histogram, and the Kolmogorov-Smirnov test was used to test for normality. Each measurement on a lower extremity was analyzed as a single observation, except when analysis of PAD cases was performed. PAD was diagnosed when the result of ABI measured by Doppler method was $\leqslant 0.9$ on one or both lower limbs. Statistical analysis was performed using Statistica 10 software (StatSoft Polska, Krakow, Poland).

\section{RESULTS}

Eighty patients ( 27 women and 53 men; mean age $70.1 \pm 9.4$ years) were enrolled in the study. Patient characteristics are presented in Table 1 . Of the 80 patients, we were unable to measure automatic ABI on the left ankle in 2 patients, and to measure systolic blood pressure on the left brachial artery in 1 patient due to previous local radiotherapy.

The Doppler method detected an $\mathrm{ABI} \leqslant 0.9$ in 56 lower extremities (35.4\% of all measurements), whereas the automated oscillometric method detected an $\mathrm{ABI} \leqslant 0.9$ in only 28 lower extremities $(17.7 \%$ of all measurements). This difference was significant $(P<0.0001)$. PAD was diagnosed by the Doppler method in $32(40 \%)$ patients out of the 80 patients analyzed. Out of the 32 patients diagnosed by the Doppler method, the automated system did not detect PAD in 10 cases (31.2\%). This difference was significant $(P<0.05)$.
Table 1 Characteristics of the study population

\begin{tabular}{lc}
\hline Mean age (age range), years & $70.1 \pm 9.4(52-88)$ \\
Sex (female/male), $n(\%)$ & $27 / 53(34 / 66)$ \\
BMI, $\mathrm{kg} \mathrm{m}^{-2}$ & $27.3 \pm 4.7$ \\
Coronary artery disease/MI/PCI/CABG, $n(\%)$ & $80(100) / 19(24) / 22(27.5) / 21(26)$ \\
Smoking active/never/past, $n(\%)$ & $27(34) / 19(24) / 34(42.5)$ \\
Previously diagnosed PAD, $n(\%)$ & $10(12.5)$ \\
Hypertension, $n(\%)$ & $63(79)$ \\
Hypercholesterolemia, $n(\%)$ & $60(75)$ \\
Diabetes mellitus, $n(\%)$ & $26(32.5)$ \\
History of atrial fibrillation, $n(\%)$ & $17(21)$ \\
History of lower extremity pain, $n(\%)$ & $34(42.5)$
\end{tabular}

Abbreviations: $\mathrm{BMI}$, body mass index; $\mathrm{CABG}$, coronary artery bypass graft; $\mathrm{PAD}$, peripheral artery disease; $\mathrm{PCl}$, percutaneous coronary intervention.

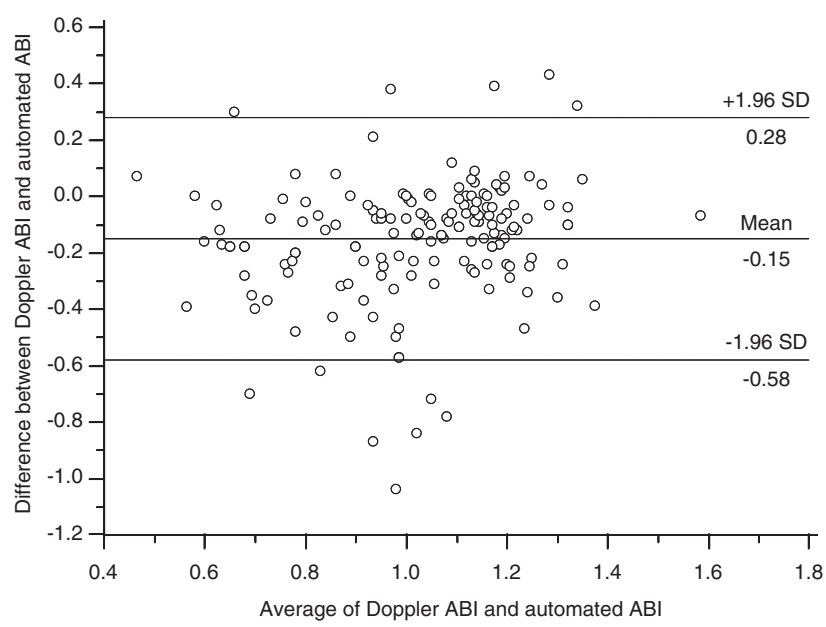

Figure 1 Bland-Altman plot of the differences in $A B I$ measurements using the Doppler and automated oscillometric methods.

There was a weak correlation between the results obtained by both methods $(r=0.51, P<0.005)$. However, the Bland-Altman plot showed poor agreement between the two methods (Figure 1). There was a trend toward larger differences between the methods in the lower range of mean $\mathrm{ABI}$ values. The mean $\mathrm{ABI}$ values obtained by the automated oscillometric method and the Doppler method were significantly different $(1.11 \pm 0.20$ vs. $0.95 \pm 0.24, P<0.00001)$ (Figure 2), and the distribution of the differences between the automated oscillometric $\mathrm{ABI}$ values and the Doppler $\mathrm{ABI}$ values did not follow a normal distribution $(P=0.027)$ (Figure 3$)$. The sensitivity of the automated oscillometric $\mathrm{ABI}$ device for detecting an $\mathrm{ABI} \leqslant 0.9$ was $46.3 \%$, whereas the specificity was $98.0 \%$. The positive and negative predictive values for diagnosing an $\mathrm{ABI} \leqslant 0.9$ using an automated method were $92.8 \%$ and $76.9 \%$, respectively.

\section{DISCUSSION}

PAD is considered to be a coronary disease equivalent that carries a risk of future cardiovascular events that is similar to that of CAD. Therefore, detection of $\mathrm{PAD}$, which remains one of the most underdiagnosed forms of atherosclerosis, is important in the general population. When PAD is detected, non-pharmacological and pharmacological interventions should be implemented to reduce the risk of cardiovascular events. 


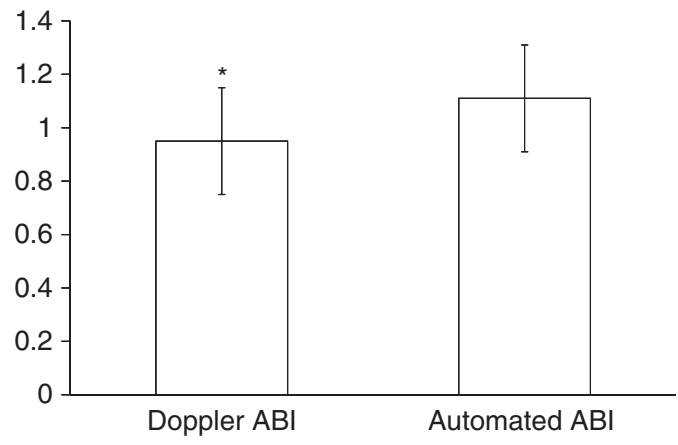

Figure 2 Mean $A B I$ measurements obtained by the Doppler and automated oscillometric methods. ${ }^{*} P<0.00001$.

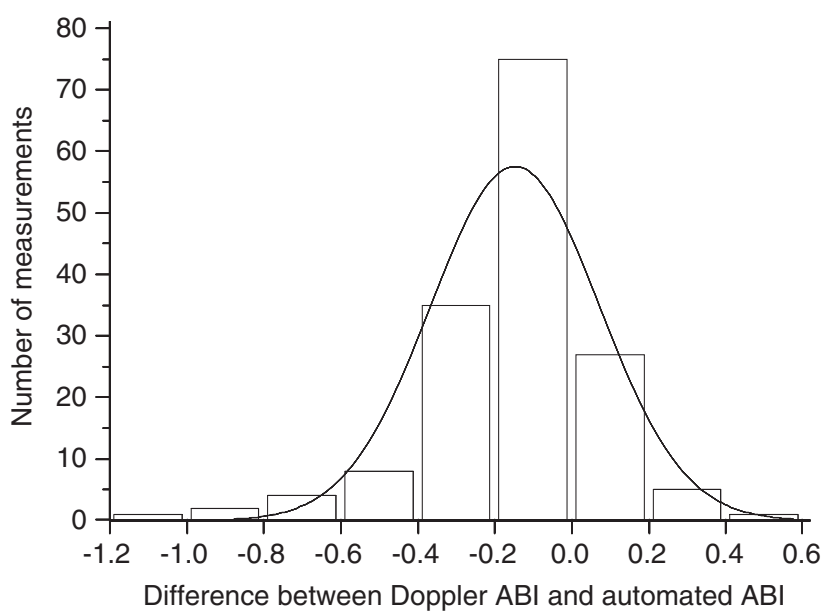

Figure 3 Non-normal distribution of the differences in $A B I$ measurements using the Doppler and automated oscillometric methods (KolmogorowSmirnow; $P=0.027$ )

It is also very important to look for PAD in individuals who are known to have atherosclerosis. First, the detection of PAD in patients with $\mathrm{CAD}$ or stroke worsens the patient's prognosis because cardiovascular mortality increases in patients with coexisting $\mathrm{CAD}$ and symptomatic or asymptomatic PAD. ${ }^{19,20}$ Second, the detection of PAD in patients with symptomatic atherosclerosis in other vascular localizations highlights the need for strict adherence to pharmacological and non-pharmacological recommendations.

The main finding of our study was that the automated oscillometric ABI measurement system did not detect almost half of all cases of lower-limb ischemia that were detected by the Doppler method in the population of subjects with CAD. This corresponded to about one-third of the patients with PAD being undetected by the automated oscillometric method. There was a significant difference in the mean $\mathrm{ABI}$ values detected by the two methods, with higher blood pressure results and ABI values obtained using the automated oscillometric system. Similar findings have been reported by other investigators. However, the detected differences between the two methods were smaller. ${ }^{12}$ In our study, we examined a high-risk population with known coronary artery atherosclerosis. In the earlier studies, only some of the subjects were known to have symptomatic atherosclerosis. This may explain, at least in part, the differences in the number of cases of undetected PAD using automated systems compared with the Doppler system in our report vs. other reports.
Table 2 Mean values for the measurements performed in this study

\begin{tabular}{lc}
\hline Automated ABI & $1.11 \pm 0.20$ \\
Doppler ABI & $0.95 \pm 0.24$ \\
Systolic blood pressure right and left arm (mm Hg) & $132.9 \pm 20 / 134.7 \pm 20$ \\
Mean EF and $n(\%)$ of $\mathrm{EF}<55 \%$ & $49.4 \pm 13.1 / 40(50)$ \\
LVDD (mm) & $49.6 \pm 8.3$ \\
Left atrium diameter (mm) & $44.8 \pm 5.4$ \\
Aorta diameter (mm) & $34.8 \pm 5.1$ \\
Intraventricular septum diameter (mm) & $12.1 \pm 2.7$ \\
Posterior wall thickness (mm) & $11.5 \pm 4.7$
\end{tabular}

Abbreviations: $A B I$, ankle-brachial index; LVDD, left ventricular diastolic diameter.

In contrast to other studies ${ }^{8,14}$ our results showed a low negative predictive value for the detection of $\mathrm{PAD}$ by the automated oscillometric system. This was the result of the significant number of PAD cases that were not diagnosed by the automated oscillometric system compared with the Doppler system.

The most important question that arises from our study is, Why did the automated oscillometric system not detect many PAD cases? One possible explanation is in line with a concept suggested by Wohlfahrt et al., ${ }^{14}$ and is related to the method of oscillometric systolic blood pressure evaluation. The oscillometric method measures the mean arterial pressure, which is described as the pressure at the point of maximal oscillations of the local artery. Systolic and diastolic blood pressures are calculated using an empirically derived algorithm. Increased arterial stiffness due to atherosclerosis may be the main causes of false results when measuring blood pressure with the oscillometric method. ${ }^{21}$

To our knowledge, none of the previous studies that compared ABI detection methods evaluated a population with such a high cardiovascular risk as the population in the current study. Here, the mean patient age was 70 years; all patients had CAD; and $75 \%$ of them had an MI, CABG and/or PCI in their medical history. Half of the subjects had reduced ejection fraction and left ventricle hypertrophy (Table 2). Importantly, one-third of the patients were active smokers. We speculate that the high-risk profile of the subjects included in the study, all of whom had clinically significant atherosclerosis, might have influenced the $\mathrm{ABI}$ measurement results obtained by the automated oscillometric device.

Our study has several limitations. We did not repeat the measurements, because they were performed during a routine examination in an echocardiographic laboratory. We were not able to assess intraobserver variability, which potentially influences the measurements. By contrast, the study results are probably typical of those obtained in a standard, busy clinical practice. Moreover, the automated oscillometric devices were designed to be used in such conditions.

The strengths of this study are that it included a coherent, high-risk patient group and that the results were obtained in a blinded fashion. A single investigator performed all of the measurements to exclude inter-observer variability.

On the basis of the results of our single-center study, we concluded that the WatchBP Office ABI system should not be used for PAD detection and screening in patients with $\mathrm{CAD}$ and cannot replace the Doppler method for monitoring patients who are at high risk of cardiovascular disease.

\section{CONFLICT OF INTEREST}

The authors declare no conflict of interest. 


\section{ACKNOWLEDGEMENTS}

This study was supported by Medical University of Warsaw.

1 Hirsch AT, Criqui MH, Treat-Jacobson D, Regensteiner JG, Creager MA, Olin JW, Krook SH, Hunninghake DB, Comerota AJ, Walsh ME, McDermott MM, Hiatt WR. Peripheral arterial disease detection, awareness, and treatment in primary care. JAMA 2001; 286: 1317-1324.

2 Lundin M, Wiksten JP, Perakyla T, Lindfors O, Savolainen H, Skytta J, Lepantalo M. Distal pulse palpation: is it reliable? World J Surg 1999; 23: 252-255.

3 Brearley S, Shearman CP, Simms MH. Peripheral pulse palpation: an unreliable physical sign. Ann R Coll Surg Eng/ 1992; 74: 169-171.

4 Fowkes FG. The measurement of atherosclerotic peripheral arterial disease in epidemiological surveys. Int J Epidemiol 1988; 17: 248-254.

5 Stoffers HE, Rinkens PE, Kester AD, Kaiser V, Knottnerus JA. The prevalence of asymptomatic and unrecognized peripheral arterial occlusive disease. Int J Epidemio 1996; 25: 282-290.

6 Fowkes FG, Housley E, Macintyre CC, Prescott RJ, Ruckley CV. Variability of ankle and brachial systolic pressures in the measurement of atherosclerotic peripheral arterial disease. J Epidemiol Community Health 1988; 42: 128-133.

7 Mohler 3rd ER, Treat-Jacobson D, Reilly MP, Cunningham KE, Miani M, Criqui MH, Hiatt WR, Hirsch AT. Utility and barriers to performance of the ankle-brachial index in primary care practice. Vasc Med 2004; 9: 253-260.

8 Beckman JA, Higgins CO, Gerhard-Herman M. Automated oscillometric determination of the ankle-brachial index provides accuracy necessary for office practice. Hypertension 2006; 47: 35-38.

9 Mundt KA, Chambless LE, Burnham CB, Heiss G. Measuring ankle systolic blood pressure: validation of the Dinamap 1846 SX. Angiology 1992; 43: 555-566.

10 Kollias A, Xilomenos A, Protogerou A, Dimakakos E, Stergiou GS. Automated determination of the ankle-brachial index using an oscillometric blood pressure monitor: validation vs. Doppler measurement and cardiovascular risk factor profile. Hypertens Res 2011; 34: 825-830.
11 Adiseshiah M, Cross FW, Belsham PA. Ankle blood pressure measured by automatic oscillotonometry: a comparison with Doppler pressure measurements. Ann R Coll Surg Eng/ 1987; 69: 271-273.

12 Hamel JF, Foucaud D, Fanello S. Comparison of the automated oscillometric method with the gold standard Doppler ultrasound method to access the ankle-brachia pressure index. Angiology 2010; 61: 487-491.

13 Aboyans V, Lacroix P, Doucet S, Preux PM, Criqui MH, Laskar M. Diagnosis of peripheral arterial disease in general practice: can the ankle-brachial index be measured either by pulse palpation or an automatic blood pressure device? Int J Clin Pract 2008; 62: 1001-1007.

14 Wohlfahrt $P$, Ingrischova $M$, Krajaoviechova A, Palous D, Dolejs ova $M$, Seidlerova J, Galovcova M, Bruthans J, Jozifova M, Adamkova V, Filipovsky J, Cifkova R. A novel oscillometric device for peripheral arterial disease screening in everyday practice. The Czech-post MONICA study. Int Angiol 2011; 30. 256-261.

15 Ramanathan A, Conaghan PJ, Jenkinson AD, Bishop CR. Comparison of anklebrachial pressure index measurements using an automated oscillometric device with the standard Doppler ultrasound technique. ANZ J Surg 2003; 73: 105-108.

16 Korno M, Eldrup N, Sillesen H. Comparison of ankle-brachial index measured by an automated oscillometric apparatus with that by standard Doppler technique in vascular patients. Eur J Vasc Endovasc Surg 2009; 38: 610-615.

17 Clairotte C, Retout S, Potier L, Roussel R, Escoubet B. Automated ankle-brachial pressure index measurement by clinical staff for peripheral arterial disease diagnosis in nondiabetic and diabetic patients. Diabetes Care 2009; 32: 1231-1236.

18 Bland JM, Altman DG. Statistical methods for assessing agreement between two methods of clinical measurement. Lancet 1986; 1: 307-310.

19 Criqui MH, Langer RD, Fronek A, Feigelson HS, Klauber MR, McCann TJ, Browner D. Mortality over a period of 10 years in patients with peripheral arterial disease. $N$ Eng/ J Med 1992; 326: 381-386.

20 Kannel WB. Risk factors for atherosclerotic cardiovascular outcomes in different arterial territories. J Cardiovasc Risk 1994; 1: 333-339.

21 van Montfrans GA. Oscillometric blood pressure measurement: progress and problems. Blood Press Monit 2001; 6: 287-290. 